\title{
Knockdown of proteolipid protein 2 or focal adhesion kinase with an artificial microRNA reduces growth and metastasis of B16BL6 melanoma cells
}

\author{
HIROKI OZAWA, YOSHIKO SONODA, TAKAHARU SUZUKI, NAOMI YOSHIDA-HOSHINA, \\ MEGUMI FUNAKOSHI-TAGO and TADASHI KASAHARA
}

Faculty of Pharmacy, Keio University, Minato-ku, Tokyo 105-8512, Japan

Received April 28, 2011; Accepted August 30, 2011

DOI: $10.3892 / 01.2011 .422$

\begin{abstract}
Proteolipid protein 2 (PLP2) promotes the metastasis of B16F10 cells in an experimental metastasis model. However, the effect of PLP2 on spontaneous metastasis has yet to be demonstrated, and whether PLP2 may become a new therapeutic target for malignant tumors is as yet unknown. In this study, PLP2 or focal adhesion kinase (FAK) microRNA-based short hairpin RNAs (miRNAs) were used as target molecules to specifically reduce the expression of PLP2 or FAK in B16BL6 cells. In vitro, the knockdown of PLP2 or FAK significantly inhibited cell proliferation, adhesion, migration and invasion. In a spontaneous metastatic tumor model using a footpad injection, the knockdown of PLP2 or FAK markedly inhibited the proliferation of the primary tumor and prevented tumor cells from invading the popliteal lymph nodes. The results indicate that downregulation of PLP2 or FAK may improve outcomes of malignant tumor therapy.
\end{abstract}

\section{Introduction}

The metastasis of malignant tumor cells from the primary tumor to distant sites in the body is a complex process that includes proliferation, adhesion, migration and invasion of metastatic tumor cells (1-3). Focal adhesion kinase (FAK) mediates numerous cell processes, including survival, migration and invasion (4). Previously, it was reported that Y925F-FAK-transfected (Y925F) melanoma cells impaired metastatic ability compared to parental B16F10 cells or vector-transfected (Vect) cells (5). Low levels of proteolipid protein 2 (PLP2) have been identified in Y925F cells by microarray analysis. Additionally, it was reported that PLP2, a four-transmembrane domain protein, has contributed to

Correspondence to: Dr Yoshiko Sonoda, Faculty of Pharmacy, Keio University, Shibakoen 1-5-30, Minato-ku, Tokyo 105-8512, Japan

E-mail: sonoda-ys@pha.keio.ac.jp

Key words: melanoma, B16BL6, metastasis, focal adhesion kinase, proteolipid protein 2, siRNA the experimental metastasis of melanoma B16F10 cells (6). Using biochemical methods, a ubiquitously expressed protein (PI3K) was identified as an associated protein of PLP2, which activates Akt, and passively enhances secretion of matrix metalloproteinase (MMP)-2 and the metastatic function of PLP2. Although these data validated the function of PLP2 in tumor metastasis, most of the data were obtained by simulating the partly metastatic process from tumor cells to distant tissues through the bloodstream. Therefore, the role of PLP2 in the entire metastatic progress from primary tumor to distant sites is unknown. Lymph node (LN) metastasis is one of the earliest features of tumor cell dissemination, and approximately $60 \%$ of metastasis is found in regional LNs in malignant melanoma. Metastasis of cancer to the regional LNs appears to reflect the biological aggressiveness of the primary tumors. The mouse melanoma cell line B16BL6 provides a convenient transplantable model, which is metastatic to LNs in the syngeneic host, C57BL/6 mice (7).

Micro RNAs (miRNAs) are the recently discovered non-coding, double-stranded RNAs that negatively regulate target gene expression. A number of studies have successfully targeted oncogenes using artificial synthetic miRNAs. Li et al (8) reported that targeting FAK via RNA interference suppressed tumor growth as well as metastasis into lung and lymph nodes in melanoma B16F10 cells. The aim of this study was to investigate the downregulation of PLP2 or FAK expression with each artificial miRNA and confirm the suppression of growth and popliteal LN metastasis of B16BL6 cells using this miRNA in C57BL/6 mice.

\section{Materials and methods}

Cell culture. The melanoma cell line B16BL6, established by Fidler (1), was obtained from Professor Yoshikazu Sugimoto of Keio University and maintained in Dulbecco's modified Eagle's medium (DMEM) supplemented with 5\% fetal bovine serum (FBS).

Gene expression analysis using GeneChip. A GeneChip analysis of empty Vect-transfected B16F10 cells and Y925F-FAK-transfected B16F10 was performed using oligonucleotide arrays (GeneChip AceGene 30K-1, containing 
30,000 mouse genes). Briefly, Cy3- or Cy5-labeled cDNAs generated from these cells were hybridized to the DNA chips, and scanned with a chip reader (Vertek, Canada). The scanned images were then processed using the MicroArray Suite (Scanalytic, Rockville, MD, USA). Data were normalized by the median of intensity of actin as a housekeeping gene.

Immunoblotting and immunoprecipitation. Immunoblotting was performed using anti-PLP2, anti-PI3K, anti-phosphoAkt, Akt and anti-phosphotyrosine. Immunoprecipitation was performed as previously described $(6,9)$.

Animals. C57BL/6J mice (6-8 weeks old) were obtained from Nippon Charles River (Tokyo, Japan). Throughout the experiments, the mice were housed in plastic cages (at $21 \pm 2^{\circ} \mathrm{C}$ ) with free access to pelleted food and water, and exposed to a $12 \mathrm{~h}$ light-dark cycle. Animal welfare and experimental procedures were performed strictly in accordance with the Guide for the Care and Use of Laboratory Animals and the related ethical regulations of the Keio University. All efforts were made to minimize the suffering of the animals and reduce the number of animals used.

Vector construction and cell transfection. Three pre-miRNA sequences were designed to target the region of the mouse PLP2 (Gene Bank accession no. NM019755) or FAK (Gene Bank accession no. NM007982) mRNA using an online tool, RNAi Designer (Invitrogen, Tokyo, Japan). Oligonucleotides encoding three different miRNAs were commercially synthesized (Invitrogen). Plasmids cloned into the pcDNA6.2-GW/ EmGFPmiR parental vector of the double-stranded DNA oligonucleotides corresponding to the three different PLP2or FAK-specific pre-miRNAs and a control sequence were obtained from Invitrogen. Plasmids were transfected into B16/ BL6 melanoma cells using Lipofectamine 2000. Following an overnight culture, the medium was exchanged to remove transfection reagents and was further incubated for $72 \mathrm{~h}$. Cells stably expressing miRNA were selected using blastcidine (40 $\mu \mathrm{g} / \mathrm{ml}$; InvivoGen, San Diego, CA, USA).

The most effective oligonucleotide sequences of the engineered pre-miRNA and adjacent flanking regions used for plasmid construction were: miRNA-FAK-3, forward: 5'-TGC TGTACAAGAGAGACTTTCAGCTAGTTTTGGCCACT GACTGACTAGCTGAATCTCTCTTGTA-3' and reverse: 5'-CCTGTACAAGAGAGATTCAGCTAGTCAGTCAGTGG CCAA A ACTAGCTGAA AGTCTCTCTTGTAC-3'; miRNA-PLP2-2, (forward): 5'-TGCTGATCTCTTGTACCA TAAGCACTGTTTTGGCCACTGACTGACAGTGCTTA GTACAAGAGAT-3' and reverse: 5'-CCTGATCTCTTGTAC TAAGCACTGTCAGTCAGTGGCCAAAACAGTGCTTA TGGTACAAGAGATC-3', negative control, forward: 5'-TGC TGAATGTACTGCGCGTGGAGACGTTTTGGCCACTG ACTGACGTCTCCACGCAGTAC-3' and reverse: 5'-CCT GAAATGTACTGCGTGGAGACGTCAGTCAGTGGCCAA AACGTCTCCACGCGC AGTACATTTC-3'.

Cell proliferation. Cell proliferation was evaluated by the MTT (tetrazolium salt) assay. Cells $\left(2 \times 10^{3}\right)$ were plated in 96 wells containing growth medium and assayed by MTT. A total of $10 \mu \mathrm{l}$ MTT [ $5 \mathrm{mg} / \mathrm{ml}$ in phosphate-buffered saline
(PBS)] was added to each well and cells were incubated at $37^{\circ} \mathrm{C}$ in a $\mathrm{CO}_{2}$ incubator. Following $3 \mathrm{~h}$, the cells were lysed using isopropanol- $\mathrm{HCl}$, and absorbance was measured at $570 \mathrm{~nm}$. All cell growth experiments were repeated at least three times.

$R T$-PCR. RNA was prepared from cells using a Qiagen Mini RNA-Easy kit (Tokyo, Japan). Reverse transcription was performed from $1 \mu \mathrm{g}$ of RNA in a $20 \mu \mathrm{l}$ reaction mixture according to the manufacturer's instructions (Takara RNA PCR kit, Ohtsu, Japan). PCR amplification and analysis were achieved using real-time PCR (Prism 7000, Applied Biosystems, Foster City, CA, USA). Primer sequences used were: PLP2, sense: 5'-GTGTGACCTGCACTCCAAGA-3' and antisense, 5'-TCAGTG GGAGCTGCTGTATG-3'; FAK, sense; 5'-CGTGAAGCCTTTTCAAGGAG-3'; and antisense, 5'-ACTGGATCTCGGGCTAGGAT-3'; MMP-2 sense: 5'-CCT GATGTCCAGCAAGTAGATG-3' and antisense: 5'-GAGACT TTGGTTCTCCAGCTTC-3'; MMP-9, sense: 5'-CCTGAT GTCCAGCAAGTAGATG-3' and antisense: 5'-GAGACTTTG GTTCTCCAGCTTC-3'.GAPDH sense: 5'-ACTCCACTCACG GCAAATTC-3' and antisense: 5'-CCTTCCACAATGCCAAA GTT-3'.

Cell adhesion assay. For the cell adhesion assay, 96-well flat-bottomed plates (ECM205 CytoMatrix screen kit, Chemicon, Temecula, CA, USA) coated with fibronectin, vitronectin, laminin and collagen I and IV were used. Cells were detached from the culture plates with a dissociation buffer, seeded at a density of $6 \times 10^{4}$ cells/well in $100 \mu \mathrm{l}$ medium and incubated at $37^{\circ} \mathrm{C}$ for $2 \mathrm{~h}$. The assay was terminated by washing the plates with PBS to remove unbound cells. The cells that remained attached to the plates were fixed and stained with $0.5 \%$ crystal violet/2\% EtOH/0.1 M borate buffer ( $\mathrm{pH} 9.0$ ). Following washing with PBS, $100 \mu 1$ solubilization buffer (1:1 mixture of $0.1 \mathrm{M} \mathrm{NaH}_{2} \mathrm{PO}_{4}, \mathrm{pH} 4.5$ and $\mathrm{EtOH}$ ) was added to the plates and measurements were taken with an ELISA reader at $540 \mathrm{~nm}$. The data were expressed as the mean absorbance of triplicate wells \pm standard deviation (SD).

Migration. Haptotaxis was assayed in triplicate using modified Boyden chambers, and cell culture inserts (Falcon) with a polyethylene terephthalate membrane $(8-\mu \mathrm{m}$ pore size, $13-\mathrm{mm}$ diameter) were used. Filters were precoated on the lower side with a $5 \mu \mathrm{g} / \mathrm{ml}$ fibronectin solution as previously described (5). Following drying, the filters were placed in the lower chamber containing $1 \%$ FBS-DMEM. Cells $\left(1 \times 10^{5}\right)$ were then added to the upper chamber supplemented with $1 \%$ FBS-DMEM and incubated at $37^{\circ} \mathrm{C}$ for $24 \mathrm{~h}$. The cells that had migrated to the underside of the insert membranes were stained with Giemsa and counted. Five fields per insert were scored, and the treatment was performed in triplicate.

Matrigel invasion assay. In vitro invasion assays were performed as previously described, with some modifications (5). A Matrigel invasion chamber (Becton-Dickinson, Franklin Lakes, NJ, USA) was used. A chemoattractant, the conditioned medium of fibroblasts, was added to the lower chamber to induce all invasion through the Matrigel. Cells were added $\left(1 \times 10^{5} /\right.$ well) to the inner chamber of the cell 
A

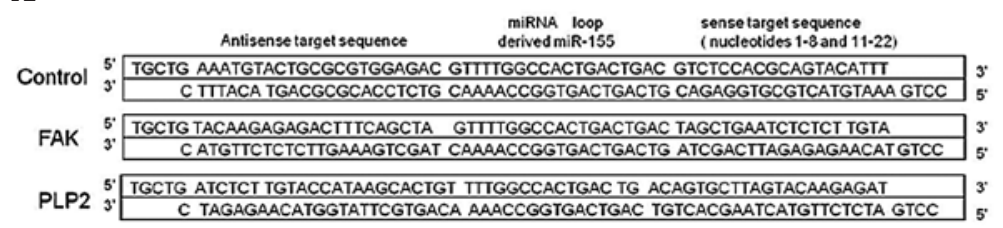

B

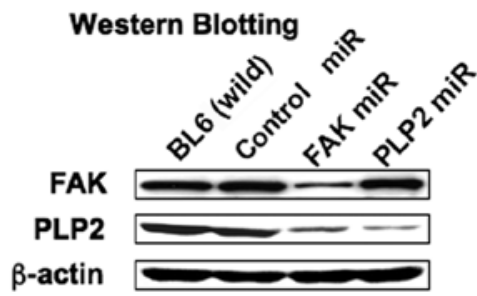

C

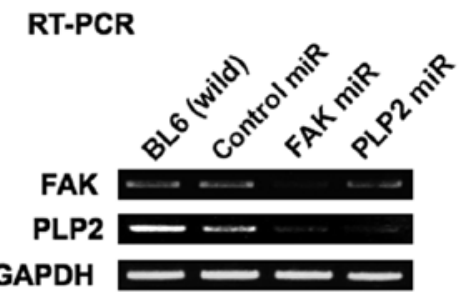

Figure 1. Downregulation of PLP2 and FAK with miRNA. (A) Pre-miRNA double-stranded oligonucleotides inserted into the miRNA pcDNA6.2-GW/ EmGFP-miR expression vector. (B) Western blotting of cells transfected with PLP2 or FAK miRNA compared to cells transfected with control miRNA. (C) RT-PCR assays for FAK and PLP2. Cells transfected with control, PLP2 or FAK miRNA were compared to the BL6 (wild).

culture insert and incubated at $37^{\circ} \mathrm{C}$ for $72 \mathrm{~h}$. The filters were fixed in $70 \%$ ethanol for $30 \mathrm{~min}$ and stained with Giemsa to quantify invasion. Non-invading cells were removed from the upper surface of the Matrigel by rubbing gently with a cottontipped applicator. Cells that had passed through the membrane were counted in five random microscopic fields of the lower filter surface.

Gelatin zymography. Cells $(\sim 70-80 \%$ confluent $)$ were washed, replenished with serum-free DMEM and incubated for $24 \mathrm{~h}$. Serum-free conditioned medium was combined with SDS buffer without heating or reduction and applied to $10 \%$ polyacrylamide gels containing $1 \mathrm{mg} / \mathrm{ml}$ of gelatin. Following electrophoresis, the gels were washed in $2.5 \%$ (v/v) Triton X-100 for $2 \mathrm{~h}$ at room temperature to remove SDS, rinsed with water, and incubated in protease buffer (50 mM Tris- $\mathrm{HCl}, \mathrm{pH} 7.5,5 \mathrm{mM} \mathrm{CaCl}_{2}, 1 \mathrm{mM} \mathrm{ZnCl}_{2}$ and $0.1 \mathrm{mM} \mathrm{NaN}_{3}$ ) for $40 \mathrm{~h}$ at $37^{\circ} \mathrm{C}$. Gels were stained with $0.5 \%$ Coomassie blue R-250 in 10\% 2-propanol and 10\% acetic acid and destained. Protease activity was visualized as a clear band.

In vivo metastasis assay. B16BL6 cells transfected with PLP2 or FAK miRNA in the exponential growth phase were harvested by trypsinization and washed twice prior to injection. Cell vitality was $>95 \%$ as determined by trypan blue dye exclusion. B16BL6 cells ( $2 \times 10^{5}$ cells in $50 \mu \mathrm{l}$ PBS) were injected into the right hind footpads of C57BL/6 mice $(100 \%$ of injected mice formed tumors). Tumor volumes were measured and calculated using the formula: $0.5236 \times \mathrm{L} 1 \times(\mathrm{L} 2)^{2}$, where L1 is the long axis and L2 is the short axis of the tumor (10). Twenty days later, mice were sacrificed and images of the right footpads and popliteal LNs were captured.

Statistical analysis. Data were expressed as the mean \pm SD. Results of in vitro experiments were analyzed using the Student's t test. For in vivo experiments, the Mann-Whitney U test was used for comparing the two groups.

\section{Results}

Suppression of PLP2 or FAK expression with each artificial miRNA. We designed three different artificial pre-miRNA sequences that targeted the PLP2 or FAK mRNA. The pre-miRNAs were cloned individually into the pcDNA6.2-GW/EmGFPmiR plasmid vector, transfected to B16BL6 cells and selected using blasticidine. The most effective sequence is shown in Fig. 1A. These cells were visualized by green fluorescence (data not shown). Basal PLP2 or FAK expression was suppressed in cells transfected with the PLP2si-2/pcDNA6.2-GW/EmGFPmiR (PLP2miR) or the FAKsi-3/pcDNA6.2-GW/EmGFPmiR (FAKmiR) plasmids individually. The suppression of PLP2 or FAK gene expression was evident compared to the cells with the control miRNA/pcDNA6.2-GW/EmGFPmiR by Western blotting and RT-PCR (Fig. 1B and C).

Effect of artificial PLP2 or FAK miRNA on the proliferation of B16BL6 cells in vitro. We determined whether PLP2 or FAK downregulation affected the tumor cell proliferation in vitro by counting cell numbers for 3 days. The results shown in Fig. 2A indicate that PLP2 or FAK downregulation markedly suppressed proliferation.

Inhibition of adhesion, migration and invasion of B16BL6 cells by PLP2 or FAK miRNA. B16BL6 cells transfected with PLP2 or FAK miRNA were investigated for their adhesion, migration and invasion abilities. PLP2 or FAK miRNA-transfected cells exhibited a significant decrease in adhesion ability to fibronectin, vitonectin, collagen IV, laminin and collagen I by more than $50 \%$ (Fig. 2B). Cells migrating to the undersurface in the migration assay were reduced by 21 and $25 \%$ (Fig. 2C), and those traversing the Matrigel in the invasion assay were reduced by 21 and $23 \%$ in B16BL6 cells transfected with PLP2 or FAK miRNA, respectively, compared to cells transfected with negative control miRNA (Fig. 2D). We examined MMP activity as a crucial step in the inva- 
A

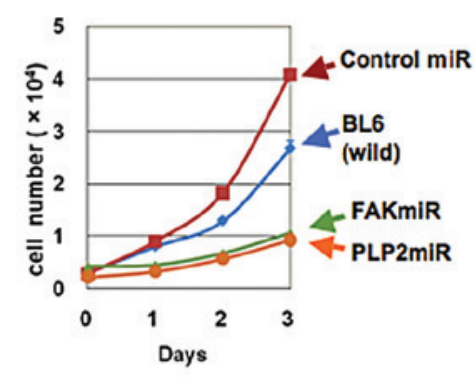

B

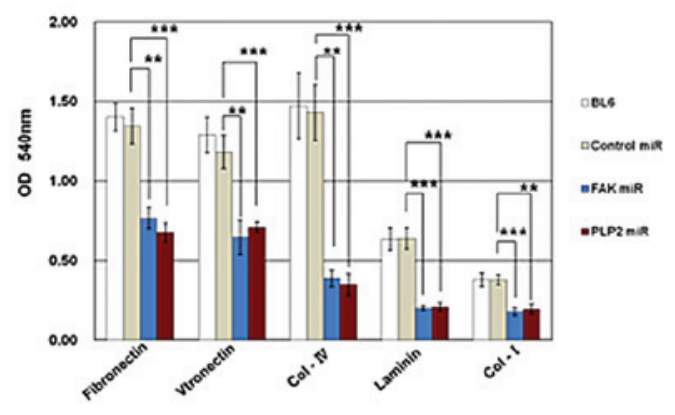

C

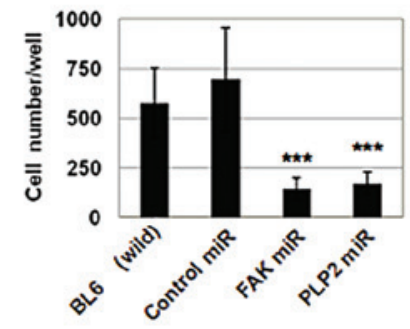

D

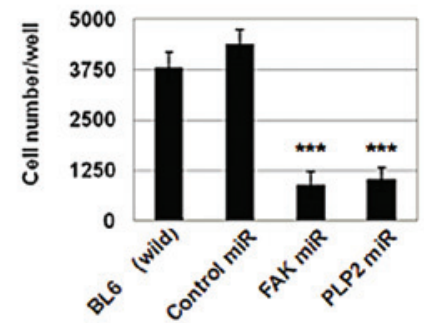

$\mathbf{E}$

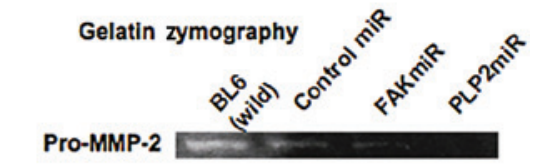

Western blotting

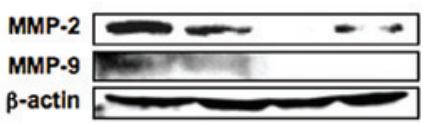

RT.PCR

MMP.2 - 2



GAPDH

Figure 2. Effects of PLP2 or FAK on proliferation, adhesion, migration and invasion. B16BL6 cells were untreated or transfected with control, PLP2 or FAK miRNA for $24 \mathrm{~h}$ and selected using blasticidin. (A) Cell proliferation: $2 \times 10^{3}$ cells per well were seeded in 96 -well plates. Cell counts were made using the MTT assay. (B) The adhesion of the parental melanoma cells or transfected melanoma cells to the extracellular matrix (fibronectin, vitronectin, laminin, and collagen I and IV) was determined as described in Materials and methods. The y-axis shows optical density following crystal violet staining of adhered cells. (C) Diagram of migrating cells. The migrating number of the cells transfected with PLP2 or FAK miRNA is shown ( ${ }^{* * *}$ P $\left.<0.001\right)$. (D) Diagram of invading cells determined by the Matrigel invasion assay. The number of invading PLP2 or FAK miRNA cells is shown $\left({ }^{* *} \mathrm{P}<0.01\right.$, $\left.{ }^{* * *} \mathrm{P}<0.001\right)$. (E) Gelatine zymography of conditioned media from cells to detect MMP-2. Cells were incubated in serum-free media for $24 \mathrm{~h}$ and conditioned media were collected for analysis. Western blotting of cells transfected with with PLP2 or FAK miRNA showed the suppression of MMP-2 and -9 compared to cells transfected with control miRNA. RT-PCR results of cells transfected with PLP2 or FAK miRNA revealed MMP-2 and -9 suppression compared to cells transfected with control miRNA.

A

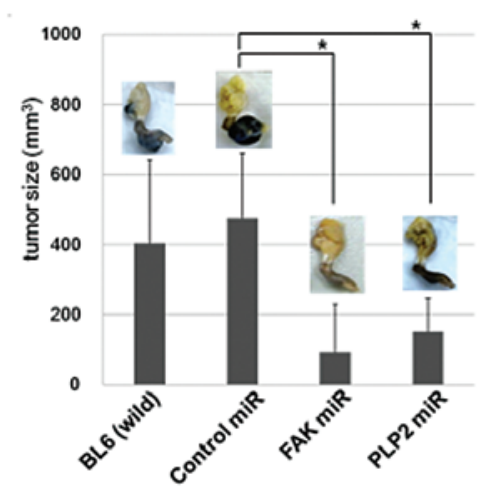

B

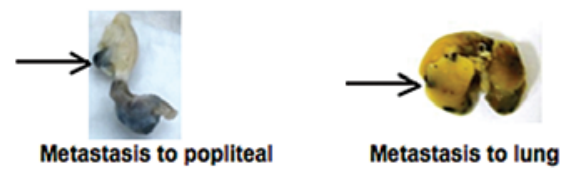

\begin{tabular}{|c|c|c|c|c|}
\hline & $\begin{array}{c}\text { BL6 (wild) } \\
(n=6)\end{array}$ & $\begin{array}{c}\text { Control miR } \\
(n=9)\end{array}$ & $\begin{array}{c}\text { FAK miR } \\
(n=9)\end{array}$ & $\begin{array}{c}\text { PLP2 miR } \\
(n=11)\end{array}$ \\
\hline $\begin{array}{c}\text { popliteal } \\
\text { lymph }\end{array}$ & $50 \%$ & $67 \%$ & $11 \%^{*}$ & $9 \%^{*}$ \\
\hline lung & $33 \%$ & $33 \%$ & $0 \%$ & $0 \%$ \\
\hline
\end{tabular}

Figure 3. Effect of PLP2 or FAK miRNA on spontaneous metastasis in B16BL6 melanoma cells in vivo. (A) B16BL6 cells $\left(20 \mu 1,2.5 \times 10^{6}\right.$ cells $\left./ \mathrm{ml}\right)$ transfected with PLP2, FAK or control miRNA were injected subcutaneously into the right footpads of C57BL/6J mice. After 20 days, the mice were sacrificed, the footpads were resected, and tumor volumes were measured ("P<0.05). (B) Metastasis frequency of B16BL6, control, PLP2 and FAK miRNA-transfected cells in popliteal lymph nodes and lungs ( $\mathrm{P}<0.05)$. 
A

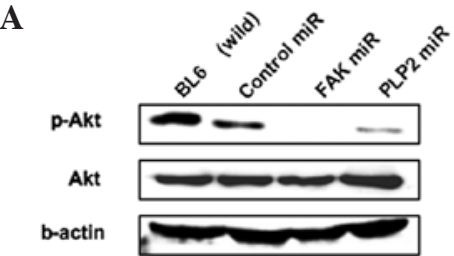

B

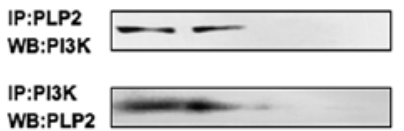

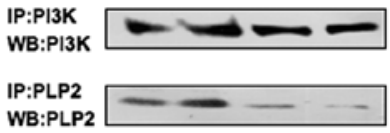

WB:PLP2

Figure 4. PLP2 and FAK miRNA blocked phosphorylation of Akt and the association with PI3K. (A) Akt phosphorylation was blocked by inhibiting PLP2 or FAK expression with artificial PLP2 or FAK miRNA. (B) Detection of the association of PI3K with PLP2 in cells transfected with PLP2, FAK or control miRNA. Lysates were prepared and immunoprecipitated with antiPLP2, and blotted with anti-PI3K and were also immunoprecipitated with anti-PI3K and blotted with anti-PLP2. Furthermore, the expression of PI3K or PLP2 in lysates is shown.

sion, which involves the degradation of extracellular matrix (ECM) components, and allows cells to efficiently traverse the basement membranes. Gelatin zymography of serumfree conditioned medium revealed that suppression of MMP activity in cells transfected with PLP2 or FAK miRNA was evident compared to cells transfected with negative control miRNA and parental cells (Fig. 2E). Suppression of MMP-2 and -9 was confirmed by Western blotting and RT-PCR (Fig. 2E). Cells transfected with PLP2 or FAK miRNA had decreased MMP-2 and -9 levels.

Inhibition of tumor growth and spontaneous metastasis by PLP2 or FAK miRNA in vivo. We then investigated whether PLP2 or FAK play a critical role in tumor formation in vivo. B16BL6 cells transfected with the PLP2 or FAK miRNA expression plasmids were subcutaneously injected into the footpads of C57BL/6J mice. PLP2 or FAK miRNA significantly suppressed tumor growth in mice, whereas control siRNA did not affect tumor growth (Fig. 3A). The mice were sacrificed and their footpads, inoculated with B16BL6 cells, were collected and images were captured. Notably, the popliteal LNs from the control miRNA-treated groups showed high ratios of visible metastases, with approximately $67 \%$ for parent and control siRNA groups. By contrast, metastasis was decreased to $11 \%$ in the popliteal LNs of PLP2 or FAK siRNA groups (Fig. 3B). Detectable metastases were observed in the lungs of the parental and control groups, but not in the PLP2 or FAK siRNA groups.

Blockade of Akt signaling by the PLP2 or FAK miRNA. Previous investigations have demonstrated that PLP2 associates with PI3K to activate Akt in B16F10 cells (6). Western blotting was performed for Akt phosphorylation to verify that the FAK-PI3K-Akt pathway was blocked due to suppression of PLP2 or FAK expression with the artificial miRNAs. The results revealed that Akt phosphorylation was detected in B16BL6 cells (Fig. 4A). Conversely, Akt phosphorylation was not detected in PLP2 or FAK miRNA-transfected cells. Furthermore, the association of PLP2 with PI3K was

confirmed. It was determined that PLP2 interacted with PI3K (Fig. 4B). The immunoprecipitation data demonstrated that this association was not detectable in the cells transfected with the miPLP2 or miFAK expression plasmids.

\section{Discussion}

We used B16BL6 cells to establish a spontaneous metastatic model and investigated its characteristics against vector-based miRNA, which was used to directly knock down the endogenous expression of PLP2 or FAK in B16BL6 cells. Tumor metastasis is a complex process that includes the detachment of cancer cells from a primary tumor, entry into the circulatory system, and adherence and migration of the tumor cells to distant sites. The first stage of tumor cell detachment from the original site depends on adhesiveness to the ECM and migration to blood capillaries and lymphatic vessels. Therefore, we examined these abilities in miPLP2- or miFAK-transfected B16BL6 cells. PLP2 or FAK knockdown by specific miRNAs suppressed B16BL6 cell proliferation in vitro, inhibited the adhesive ability of B16BL6 cells to the ECM, such as fibronectin, and inhibited migration and invasion ability in the transwell-based assay. The adhesion, migration and invasion ability of tumor cells may be regulated by multiple signaling cascades, including integrin-mediated signaling, mitogen-activated protein kinase signaling and cytoskeletal reorganization. This hypothesis is substantiated by the observation that PLP2 directly interacted with PI3K and activated the Akt signaling pathway. These findings further confirm that PLP2 and/or FAK play a key role in the acquisition of metastatic potential by tumor cells.

Experimentally passive models, which mimic the metastatic events after tumor cells enter the circulatory system by injecting tumor cells to tail veins, have been used to investigate the function of PLP2 in vivo, but the role of PLP2 in cancer cell detachment from the original site, such as adhesion to the ECM, digestion of the matrix and intravasation, has not been explored. A previous study indicated that PLP2 transfectants attach well to fibronectin, a common matrix of adhesion and migration assays (6). The present study reconfirmed those observations and showed that PLP2 or FAK miRNA may inhibit the adhesion, migration and invasion ability of B16BL6 cells in vitro. Additionally, PLP2 or FAK miRNA treatment markedly reduced the metastatic ability of B16BL6 cells to migrate to popliteal LNs, and no visible metastatic sites were detected in that group. These results suggest that the in vivo proliferation of B16BL6 cells at the primary tumor site and the development of macroscopic metastases were highly dependent on the PLP2 or FAK expression level. Untreated B16BL6 cells, with normal endogenous PLP2 expression, showed more apparent metastasis to popliteal LNs than that of PLP2 miRNA-treated cells, whereas $33 \%$ of metastatic sites were visible in the lung.

The data show the substantial role that PLP2 and FAK play in spontaneous metastasis in vivo. Further studies are necessary to reveal whether PLP2 regulates the expression of these factors in the process of popliteal LN metastasis. B16BL6 cells treated with PLP2 miRNA as well as FAK miRNA showed slow growth compared with those treated with negative miRNA in primary tumors in vivo. 
In conclusion, the present study reproduced the role of PLP2 or FAK in the entire process of spontaneous metastasis from primary tumor to regional $\mathrm{LN}$. We also found that specifically reduced PLP2 expression inhibited the growth of B16BL6 cells in vivo and prevented detectable metastasis from primary tumors by decreasing adhesive ability in the ECM, such as fibronectin and laminin, and by reducing the migratory ability of B16BL6 cells. We found that PLP2 or FAK played a more pivotal role in tumor formation and metastasis in B16BL6 cells. The current results extend potential therapeutic applications of PLP2 or FAK miRNA to the clinical treatment of malignant tumors.

\section{Acknowledgements}

We thank Erika Suzuki and Saori Kato for their technical assistance. This study was supported in part by a grant (no. 20590069) from the Ministry of Education, Culture, Sports, Science and Technology of Japan.

\section{References}

1. Fidler IJ: The pathogenesis of cancer metastasis the 'seed and soil' hypothesis revisited. Nat Rev Cancer 3: 453-458, 2003.

2. MacDonald IC, Groom AC and Chambers AF: Cancer spread and micrometastasis development: quantitative approaches for in vivo models. Bioessays 24: 885-893, 2002.
3. Hendrix MJ, Seftor EA, Seftor RE, Kasemeier-Kulesa J, Kulesa PM and Postovit LM: Reprogramming metastatic tumour cells with embryonic microenvironments. Nat Rev Cancer 7: 246-255, 2007.

4. Hanks SK, Ryzhova L, Shin NY and Brábek J: Focal adhesion kinase signaling activities and their implications in the control of cell survival and motility. Front Biosci 8: 982-996, 2003.

5. Kaneda T, Sonoda Y, Ando K, Suzuki T, Sasaki Y, Oshio T, Tago $\mathrm{M}$ and Kasahara T: Mutation of Y925F in focal adhesion kinase (FAK) suppresses melanoma cell proliferation and metastasis. Cancer Lett 270: 354-61, 2008.

6. Sonoda Y, Warita M, Suzuki T, Ozawa H, Fukuda Y, Funakoshi-Tago $\mathrm{M}$ and Kasahara T: Proteolipid protein 2 is associated with melanoma metastasis. Oncol Rep 23: 371-6, 2010.

7. Raz A, Bucana C, McLellan W and Fidler IJ: Distribution of membrane anionic sites on B16 melanoma variants with differing lung colonising potential. Nature 284: 363-364, 1980.

8. Li S, Dong W, Zong Y, Yin W, Jin G, Hu Q, Huang X, Jiang W and Hua ZC: Polyethylenimine-colexed plasmid particles targeting focal adhesion kinase function as melanoma tumor therapeutics. Mol Ther 15: 515-23, 2007.

9. Sonoda Y, Watanabe S, Matsumoto Y, Aizu-Yokota E and Kasahara T: FAK is the upstream signal protein of the phosphatidylinositol 3-kinase-Akt survival pathway in hydrogen peroxide-induced apoptosis of a human glioblastoma cell line. J Biol Chem 274: 10566-70, 1999.

10. Qian F, Li YP, Sheng X, Zhang ZC, Song R, Dong W, Cao SX Hua ZC and Xu Q: PRL-3 siRNA inhibits the metastasis of B16-BL6 mouse melanoma cells in vitro and in vivo. Mol Med 13: 151-9, 2007. 